\title{
Introduction to the special issue of the multimedia tools and applications journal on events in multimedia
}

\author{
Ansgar Scherp • Ramesh Jain • Mohan S. Kankanhalli
}

Published online: 18 May 2011

(C) Springer Science+Business Media, LLC 2011

Humans think in terms of events and entities. Events provide a natural abstraction of happenings in the real world. The concept of events has a long history in foundational sciences such as philosophy and linguistics. After first developing object-based and entitybased approaches, computer science research is now addressing the concept of events and building many applications that consider events at least as important as objects. Consequently, we find many different solutions and approaches for modeling, detecting, and processing events. In addition, we find different applications that are based on events and that make use of events.

This special issue of Springer's Multimedia Tools and Applications journal on Events in Multimedia focuses on the detection, modeling, and processing of events and applications that make use of events in the context of multimedia data. The first paper "Ice hockey shooting event modeling with mixture hidden Markov model" presents a framework for analyzing events in ice hockey videos using mixture hidden Markov model (HMM). Ice hockey videos are hard to analyze due to the homogeneity of the video material. The paper shows that mixture HMM is a suitable approach for event detection in ice hockey videos with high accuracy.

The second paper, "Event retrieval in video archives using rough set theory and partially supervised learning" presents a query-by-example approach where users provide example event shots in order to retrieve event shots from the TRECVID 2009 video data set. As a single model cannot be used for retrieval, different classifiers are combined into rules using rough set theory. Counter examples, which are necessary for applying rough set theory, do not need to be provided by the users but are compensated by applying a partially supervised learning method.

\footnotetext{
A. Scherp $(\square)$

University of Koblenz-Landau, Koblenz, Germany

e-mail: scherp@uni-koblenz.de
}

R. Jain

University of California, Irvine, CA, USA

M. S. Kankanhalli

National University of Singapore, Singapore, Singapore 
The paper "Abstracting and reasoning over ship trajectories and web data with the Simple Event Model (SEM)" aims at bridging the gap between low-level features and semantics by representing the knowledge at different level of abstraction. To this end, the Simple Event Model (SEM) is proposed and applied for situation awareness in the domain of maritime safety and security. The SEM allows representation of simple behavior events that are captured from processing low-level ship trajectory data. Further aggregation of the simple behavior events and the application of deduction rules and spatial proximity reasoning allow extraction of more abstract events.

Finally, the paper on "Unifying and targeting cultural activities via events modeling and profiling" presents a framework for aggregating, enriching, recommending, and distributing cultural events based on the users' interests. The goal is to provide a usable recommendation platform that allows its users to access useful event information beyond mere retrieval of event information. The aggregated events are published as Linked Open Data and thus can be connected with other event sources on the web.

We thank all the authors who have contributed to this special issue of the Multimedia Tools and Applications journal by submitting their work. Our gratitude also goes to the reviewers for spending their precious time on reading the articles and providing valuable feedback to the authors. Finally, our thanks go to the Editor-in-Chief of the journal, Professor Borko Furht, for accepting and supporting our special issue on Events in Multimedia. Thank you for providing us the platform to present recent developments for detecting, modeling, and processing of events in multimedia and applications that make use of such events. We would also like to take the opportunity to thank the staff at Springer for their effort in putting the pieces for the special issue together and helping us in the production process. We hope that the special issue will be of benefit for the research community on Events in Multimedia.

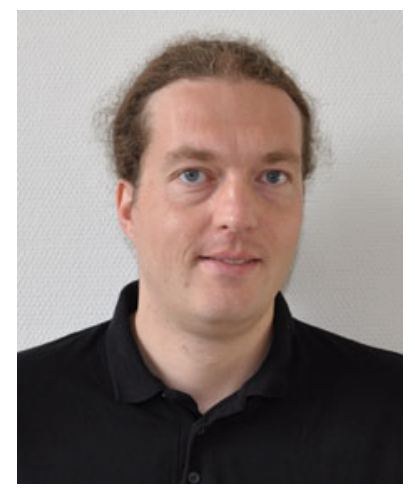

Ansgar Scherp is Juniorprofessor at the University of Koblenz-Landau, Germany and leading the focus group on Interactive Web at the Institute for Web Science and Technologies. He has received his $\mathrm{PhD}$ at the University of Oldenburg, Germany with distinction in 2006. He has been EU Marie Curie Fellow at the Donald Bren School of Information and Computer Sciences, University of California, Irvine, USA between 2006 and 2008. He serves as Program Committee member for conferences including ACM Multimedia, Multimedia Modeling, and International Semantic Web Conference and journals including IEEE Multimedia, Springer's Multimedia Systems, ACM Transactions on Multimedia Computing Communications and Applications, and Journal of Web Semantics. Mr. Scherp co-authored over 50 peer-reviewed scientific publications. Mr. Scherp is co-founder of the startup company Kreuzverweis Solutions GmbH providing a media management solution based on semantic technologies. His interests are the formal modeling of events and event-relations and applying events in the context of semantic computing and mobile computing. 


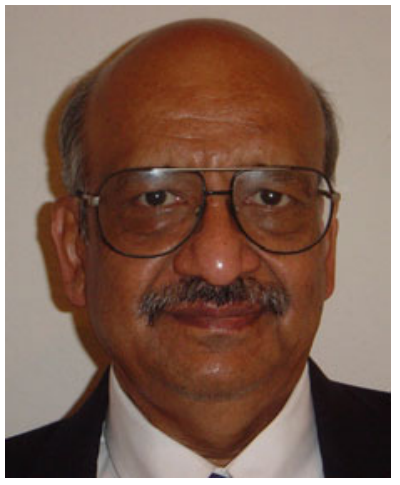

Ramesh Jain is an educator, researcher, and entrepreneur. Currently he is the Donald Bren Professor in Information \& Computer Sciences at University of California, Irvine. He is also a Distinguished Visiting Professor at National University of Singapore. Ramesh was the founding Editor-in-Chief of IEEE Multimedia magazine and serves on the editorial boards of several journals in multimedia, information retrieval and image and vision processing. He served as the Chairman of ACM SIG Multimedia. He has coauthored more than 300 research papers, co-authored and co-edited several books. He is a Fellow of ACM, IEEE, AAAI, IAPR, and SPIE. Ramesh Jain has a long term expertise in event detection and event modeling. Is also co-founder of three companies several start-up companies that deal with events, managed them in initial stages, and then turned them over to professional management. Currently, he serves as advisor to many companies.

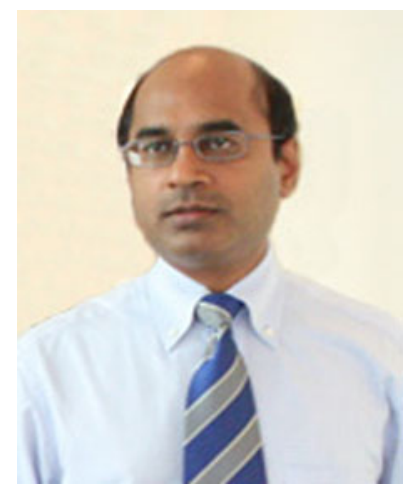

Mohan S. Kankanhalli is Professor at the Department of Computer Science at the National University of Singapore (NUS). He is also the Associate Provost for Graduate Education at NUS. Mohan obtained his bachelor's in EE from IIT Kharagpur and MS \& PhD from the Rensselaer Polytechnic Institute. He is actively involved in the organization of many major conferences in the area of Multimedia. He is on the editorial boards of several journals including the ACM Transactions on Multimedia Computing, Communications, and Applications, Springer Multimedia Tools \& Applications Journal, Springer Multimedia Systems Journal, Pattern Recognition Journal and the LNCS Transactions on Data Hiding and Multimedia Security. He has a particular interest in event detection and event mining from multimodal data. A lot of this work is done in the context of surveillance and monitoring applications. 